EPJ Web of Conferences 75, 03001 (2014)

DOI: $10.1051 /$ epjconf/ 20147503001

(C) Owned by the authors, published by EDP Sciences, 2014

\title{
Magnetism in the interface of $\mathrm{Co} / \mathrm{CoO}$
}

\author{
D. Tzeli ${ }^{1, \mathrm{a}}$, A. Morphis ${ }^{1}$, J. A. Blackman ${ }^{2}$, and K. N. Trohidou ${ }^{1}$ \\ ${ }^{1}$ IAMPPNM, Department of Materials Science, NCSR Demokritos, Athens, Greece \\ ${ }^{2}$ Department of Physics, University of Reading, Reading RG6 6AF, United Kingdom
}

\begin{abstract}
We have employed first-principles calculations to study the magnetic properties of the binary system Co-O. Two types of calculations were carried out: i. Co-O clusters of 13, 14 and 19 atoms and ii. $\mathrm{Co} / \mathrm{CoO}$ bilayer. The $\mathrm{Co} / \mathrm{CoO}$ bilayer forms a ferromagnetic-antiferromagnetic interface. The geometrical structures of the clusters and of the $\mathrm{Co} / \mathrm{CoO}$ bilayer were optimized via ab initio collinear and non-collinear calculations. The spin-orbit interaction was included, too. We found that the addition of the $\mathrm{O}$ atom in the Co cluster leads to an increase of the $\mu_{\mathrm{Co}}$ values of O's adjacent Co atoms. At the bilayer, the Co atoms in the interface have enhanced magnetic moment compared to the corresponding values of the bulk Co in agreement with the experiment.
\end{abstract}

\section{Introduction}

Magnetic transition metal nanoclusters are receiving considerable attention due to the huge number of the current and potential application in many areas of technology e.g. from nanomedicine to magnetic storage devices and logic processing devices [1]. Many of their properties differ from the bulk materials due to their reduced size, for example they present unique geometric structures [2] and unusually high magnetic moments [34]. Similarly, transition-metal oxides, such as $\mathrm{CoO}$, have attracted the interest due to their unique structural, electrical, optical, and magnetic properties and to their numerous magnetic, magneto-optical and magnetoelectrical applications [5-6].

Therefore, the study of magnetism in clusters of transition metal atoms [7], in bimetallic magnetic interfaces [8] and in bilayers that form a ferromagnetic/antiferro-magnetic interface [9-10] is of immense interest. While the bulk $\mathrm{Co}$ and $\mathrm{CoO}$ material and their magnetic properties have been thoroughly studied experimentally, see for instance [10-20], and theoretically, via density functional theory (DFT) and Monte Carlo simulations, see for instance [21-24], attention has not been given yet for small clusters of Co$\mathrm{O}$. Moreover, while the $\mathrm{Co} / \mathrm{CoO}$ system has been widely studied experimentally [9, 25-27], there is not any theoretical study.

In the present study, we carried out ab initio calculations on two different systems. At the first part, we study the $\mathrm{Co}_{x}$ and $\mathrm{Co}_{x-1} \mathrm{O}$ clusters, where $x=13,14$, and 19. Our aim is to calculate the magnetic moments of the atoms and to study how the presence of the $\mathrm{O}$ atom

\footnotetext{
${ }^{\mathrm{a}}$ Corresponding author: dtzeli@ims.demokritos.gr
}

affects the magnetic moment of the Co atoms in the $\mathrm{Co}_{\mathrm{x}-1}$ clusters. At the second part of the present work, preliminary calculations on the $\mathrm{Co} / \mathrm{CoO}$ bilayer that forms a ferromagnetic/antiferro-magnetic interface are reported. Our aim is to study the magnetic moments of the $\mathrm{Co}$ atoms of the $\mathrm{Co}$ and $\mathrm{CoO}$ layers in the interface.

\section{Computational details}

DFT calculations were carried out on $\mathrm{Co}_{x}$ and $\mathrm{Co}_{x-1} \mathrm{O}$ clusters, where $x=13,14$, and 19 , and on $\mathrm{Co} / \mathrm{CoO}$ bilayer. The Co layer has an fcc structure, while $\mathrm{CoO}$ a rock salt (RS) structure and the two surfaces are touching via the (001) orientation. All computations were performed using the Vienna ab initio simulation package (VASP4.6) $[28,29]$. The $4 s^{2} 3 d^{7}$ and $2 s^{2} 2 p^{4}$ electrons of $\mathrm{Co}$ and $\mathrm{O}$ are treated as valence. The electronic oneparticle wave functions are expanded in a basis set of plane waves with kinetic energies up to $600 \mathrm{eV}$. The projector-augmented wave (PAW) method is employed to describe the wave functions in the core regions. The GGA exchange-correlation potential of the Perdew-Wang 91 form, [30] as implemented in the VASP, were employed. Given the limitations of the GGA to describe systems with strongly correlated $d$ electrons, a $d$ - $d$ intraatomic Coulomb energy $U$ is introduced. Thus, all present calculations are $\mathrm{GGA}+\mathrm{U}$, based on the scheme of Dudarev et al. [31]. The Coulomb $U$ and exchange J parameters are chosen to be $\mathrm{U}=5, \mathrm{~J}=1$, as these values correctly reproduce experimental data of the $\mathrm{Co}$ and $\mathrm{CoO}$ bulk material, see below. All geometries of the clusters were fully optimized via collinear calculations. In some cases non-collinear calculations were carried out and the

This is an Open Access article distributed under the terms of the Creative Commons Attribution License 2.0, which permits unrestricted use, distribution, and reproduction in any medium, provided the original work is properly cited. 
spin-orbit coupling was included, too. In the case of the bilayer, its geometry was optimized via collinear calculations. All Hellman-Feynman forces for each atom are $<0.005 \mathrm{eV} / \AA$. Only the $\Gamma$ point was sampled in the calculations of the clusters, while the Brillouin zone for the bilayer is sampled by a mesh of $9 \times 9 \times 1$ MonkhorstPack k-grid. For the calculations of the $\mathrm{Co}(\mathrm{fcc})$ and $\mathrm{CoO}(\mathrm{RS})$ bulk materials unit shells up to 16 atoms were used and both the $9 \times 9 \times 9$ and $11 \times 11 \times 11$ Monkhorst-Pack k-grid were applied.

\section{Results and Discussion}

\subsection{Bulk Co and CoO}

The Coulomb $\mathrm{U}$ and exchange $\mathrm{J}$ parameters are chosen to be $\mathrm{U}=5, \mathrm{~J}=1$, as these values correctly reproduce most of the experimental data of the $\mathrm{Co}$ and $\mathrm{CoO}$ bulk material, i.e., the magnetic moments of Co atoms $\left(\mu_{\mathbf{C o}}\right)$, the lattice constants (a), and the band gap of $\mathrm{CoO}$ (RS). Our calculated data along with the experimental values are presented in Table 1 . The $\mathrm{CoO}(\mathrm{RS})$ has been calculated as an antiferromagnetic material with a type-II AFM ordering, where the magnetic moments on $\mathrm{Co}^{2+}$ ions within the same (111) plane are parallel and antiparallel to each other between the adjacent (111) planes. The type-II AFM ordering has been has been confirmed both experimentally [12-13] and theoretically [21].

Table 1. Lattice constants ( $\mathrm{a}$ in $\AA$ ), magnetic moments of Co atoms $\left(\mu_{\mathrm{Co}}\right.$ in $\left.\mu_{\mathrm{B}}\right)$, charge of $\mathrm{Co}$ and $\mathrm{O}\left(\mathrm{q}_{\mathrm{co}}\right.$ and $\mathrm{q}_{\mathrm{o}}$ in $\left.\mathrm{e}^{-}\right)$and the band gap (in eV) of $\mathrm{Co}(\mathrm{fcc})$ and $\mathrm{CoO}(\mathrm{RS})$ bulk materials.

\begin{tabular}{|c|c|c|c|c|}
\hline & \multicolumn{2}{|c|}{ Co(fcc) } & \multicolumn{2}{c|}{ CoO(RS) } \\
\hline & GGA+U & expt & GGA+U & expt \\
\hline $\mathbf{a}$ & 3.56 & $\begin{array}{c}3.548- \\
3.573^{\mathrm{a}}\end{array}$ & 4.27 & $4.2667^{\mathrm{c}}$ \\
\hline $\boldsymbol{\mu}_{\mathbf{C o}}$ & 1.78 & $1.751^{\mathrm{b}}$ & 2.71 & $\begin{array}{c}3.35^{\mathrm{d}} \\
3.8,^{\mathrm{e}} \\
3.98(6)^{\mathrm{f}}\end{array}$ \\
\hline $\mathbf{q}_{\mathbf{C o}} / \mathbf{q}_{\mathbf{o}}$ & 8.27 & & $7.81 / 4.94$ & \\
\hline Band gap & & & 2.69 & $2.5 \pm 0.3^{\mathrm{g}}$ \\
\hline
\end{tabular}

${ }^{a}$ The lattice constant depends on temperature, Ref. 14-15. ${ }^{\mathrm{b}}$ Ref. 16. ${ }^{\mathrm{c}}$ Ref. 17. ${ }^{\mathrm{d}}$ Ref. $18 .{ }^{\mathrm{e}}$ Ref. $12 .{ }^{\mathrm{f}} \operatorname{Ref} 19 .{ }^{\mathrm{g}}$ Ref 20.

We should note that for the bulk Co(fcc), both GGA and GGA $+U$ methods, computed the same a and $\mu_{\mathrm{Co}}$ values, in complete agreement with the experimental data. For the bulk $\mathrm{CoO}(\mathrm{RS})$, the GGA method predict correctly only the lattice constant, while the GGA+U method predict correctly both the lattice constant and the band gap, but there is a deviation of the $\mu_{\mathrm{Co}}$ values from the experimental ones. The present computed $\mu_{\text {Co }}$ value for the bulk $\mathrm{CoO}$ are in complete agreement with previous calculated values 2.77 [23], 2.4-2.7 [24], 2.412.67 [21]. However, all underestimate the experimental findings, where a value ranging from 3.35 to $3.98(6) \mu_{\mathrm{B}}$ is predicted. The underestimation of the theoretical results comes from the orbital contribution to the magnetic moment, which is argued to be comparable large [24]. Calculations on $\mathrm{CoO}_{6}$ octahedral complex predict an orbital moment of $1.39 \mu_{\mathrm{B}}$ and spin moment of $2.62 \mu_{\mathrm{B}}$, the same value with calculated magnetic moments of the bulk, see Table 1 and references [21, 23, 24]. Nevertheless, here, we study how the $\mu_{\mathrm{Co}}$ magnetic moments are changed from $\mathrm{Co}_{\mathrm{x}}$ clusters to $\mathrm{Co}_{\mathrm{x}-1} \mathrm{O}$ and in the interface of $\mathrm{Co} / \mathrm{CoO}$ bilayers. Thus, even though we have some differences with the experimental values, we are interested for the relative changes. Finally, we have to add that the oxygen atoms in $\mathrm{CoO}$ carry no magnetic moment.

\section{2 $\mathrm{Co}_{x}$ clusters, $x=13,14$ and 19}

We computed the three clusters shown in figure 1 containing 13, 14 and 19 atoms, respectively. Their equilibrium structures are an icosahedron, a cube and a double icosahedron. The magnetic moments of the Co atoms are given in Table 2 and they are enhanced over the bulk values, see Table 1 , in agreement with the experimental findings [32-34], where values of $2.08 \pm 0.20$ $\mu_{\mathrm{B}}$ are measured for clusters of 20-200 atoms [32]. In the case of the cluster $\mathrm{Co}_{14}$, which has the structure of the bulk $\mathrm{Co}(\mathrm{fcc})$, our computations shows that the crystal directions in order of increasing difficulty of magnetization axis is [110], [100], [111], while in the bulk we found that it changes to [111], [110], [100] in complete agreement with the experimental findings [10]. The difference in the order of the easy magnetization axis is attributed to the finite size effects of the system that affect their magnetic properties.
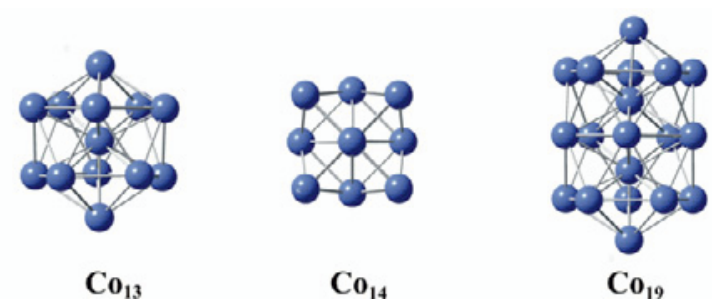

Fig. 1. $\mathrm{Co}_{13}, \mathrm{Co}_{14}$, and $\mathrm{Co}_{13}$ clusters.

Table 2. Calculated GGA+U magnetic moments of the Co atoms in $\mathrm{Co}_{\mathrm{x}}$ and $\mathrm{Co}_{\mathrm{x}-1} \mathrm{O}$ clusters.

\begin{tabular}{|c|c|c|}
\hline & $\mathbf{C o}_{\mathbf{x}}$ & $\mathbf{C o}_{\mathbf{x}-\mathbf{1}} \mathbf{O}$ \\
\hline $\mathbf{x}=\mathbf{1 3}$ & $1.8-2.2$ & $1.9-2.3$ \\
\hline $\mathbf{x}=\mathbf{1 4}$ & $1.9-2.0$ & $1.7-2.1$ \\
\hline $\mathbf{x}=\mathbf{1 9}$ & $1.9-2.0$ & $1.9-2.4$ \\
\hline
\end{tabular}

\section{$3.3 \mathrm{Co}_{\mathrm{x}-1} \mathrm{O}$ clusters, $\mathrm{x}=13,14$ and 19}

Generally, the systematic search for the most stable structures of a given cluster is a very demanding task as it involves the scan of the potential energy surface. Specifically for a cluster having transition metals, it is even more difficult because of: a. the dominant role of 
the d-electrons which are relatively strong localized, they have directional bonding and high density of states around the Fermi energy; b. the presence of many closelying electronic configurations with different spin, c. the need of an accurate treatment of the exchange-correlation effects within the DFT methodology [35]. The task becomes even more demanding when someone has to calculate magnetic structures.
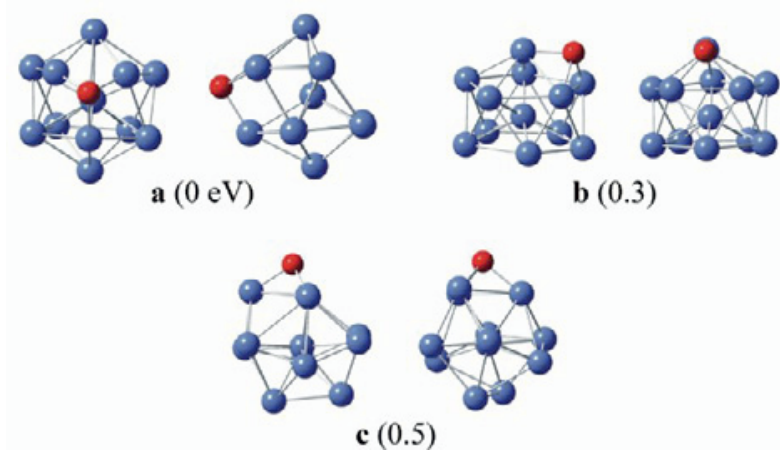

Fig. 2. $\mathrm{Co}_{12} \mathrm{O}$ clusters: Three magnetic structures viewed from two different angles. Energy differences from the lowest in energy magnetic structure in parentheses (Co: light balls (blue online) and $\mathrm{O}$ : dark grey (red online)

In the present calculations of the $\mathrm{Co}_{x-1} \mathrm{O}$ clusters, initially we replaced a $\mathrm{Co}$ atom with an $\mathrm{O}$ atom. There are 3,2 and 4 inequivalent sites at the three $\mathrm{Co}_{13}, \mathrm{Co}_{14}$, and $\mathrm{Co}_{13}$ clusters, respectively. Then, the clusters were optimized and stable magnetic structures were obtained.
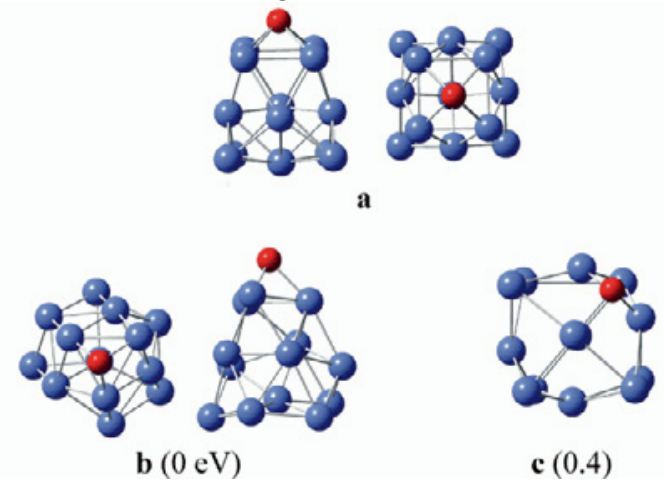

Fig. 3. $\mathrm{Co}_{13} \mathrm{O}$ clusters: $\mathbf{a}$ is nomagnetic structure; $\mathbf{b}$ and $\mathbf{c}$ are magnetic structures. The $\mathbf{a}$ and $\mathbf{b}$ magnetic structures viewed from two different angles. Energy differences from the lowest in energy magnetic structure in parentheses (Co: light balls (blue online) and O: dark grey (red online)

The lowest calculated magnetic structures are depicted in figures 2-4 along with the energy differences from the lowest in energy magnetic structure. Three minima are given for the $\mathrm{Co}_{12} \mathrm{O}$ cluster, two for the $\mathrm{Co}_{13} \mathrm{O}$ cluster and 5 for the $\mathrm{Co}_{18} \mathrm{O}$ cluster. In the lowest stable magnetic structures, the $\mathrm{O}$ atom prefers to be in an outer position rather than in the center of the cluster. Thus, while for the $\mathrm{Co}_{12} \mathrm{O}$ cluster the substitution of the inner $\mathrm{Co}$ atom with an $\mathrm{O}$ atom does not lead to a stable magnetic structure, and geometry optimization results in being the $\mathrm{O}$ atom in an external position, for the $\mathrm{Co}_{18} \mathrm{O}$ cluster, results in a stable magnetic structure (figure 4, e structure), but it is located very high, at $3.3 \mathrm{eV}$ above the lowest in energy structure. For the case of the $\mathrm{Co}_{12} \mathrm{O}$ a symmetric no magnetic structure (a) has been calculated, see figure 3 , but when magnetic calculations were carried out, this structure was not stable and it was turned out to the stable magnetic structure $\mathbf{b}$, where the symmetry is broken. Finally, for the case of the $\mathrm{Co}_{18} \mathrm{O}$, many minima structures were obtained. Three of them (a-c) are practically energetically degenerate, see figure 4 .
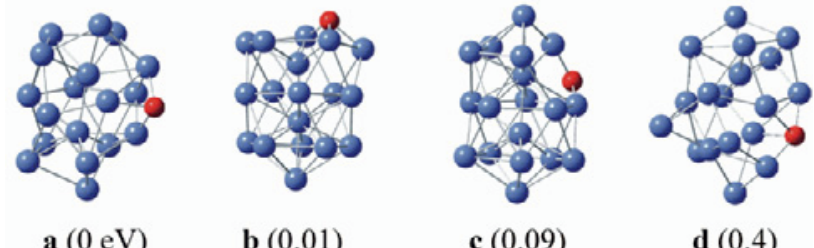

c $(0.09)$

d $(0.4)$

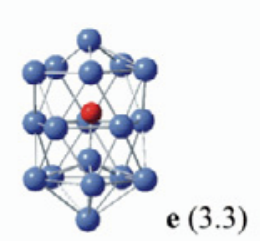

Fig. 4. $\mathrm{Co}_{18} \mathrm{O}$ clusters: Energy differences from the lowest in energy magnetic structure in parentheses (Co: light balls (blue online) and O: dark grey (red online)

The magnetic moments of the Co atoms are presented in Table 2. Similar results for all magnetic minima structures were obtained regarding the magnetic moments of the Co atoms. The replacement of a Co atom with an $\mathrm{O}$ atom results in a small increase of the average magnetic moment of the Co atoms of the cluster. The largest increase is observed for O's adjacent Co atoms. Moreover, very small less than $0.1 \mu_{\mathrm{B}}$ magnetic moments are induced in the $\mathrm{O}$ atoms. We should note that we relaxed some of the calculated structures with noncollinear initial spin structures and collinear magnetic structures were obtained, showing that the preference of the noncollinearity plays no important role here. However, furthermore calculations will be conducted to confirm this result.

\section{$3.4 \mathrm{Co} / \mathrm{CoO}$ bilayers}

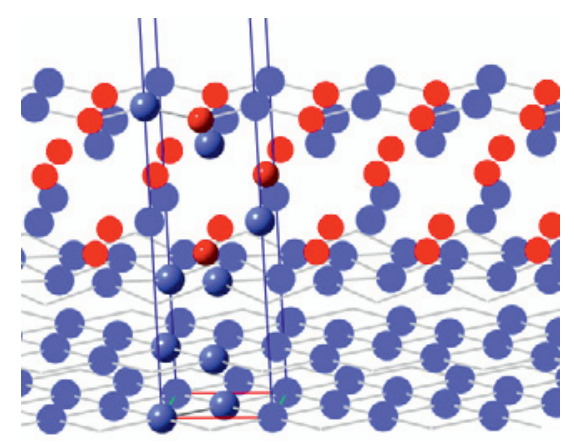

Fig. 5. $\mathrm{Co} / \mathrm{CoO}$ bilayer: The super-cell with its 12 atoms which are shown as balls. The atoms of the adjacent super-cells are dull. (Co: light balls (blue online) and O: dark grey (red online).

Preliminary magnetic calculations on the interface of the $\mathrm{Co} / \mathrm{CoO}$ bilayer were carried out. Our model consists of 6 layers, 3 layers of $\mathrm{Co}$ and 3 layers of $\mathrm{CoO}$, see figure 6 . 
In total, the super-lattice has 9 atoms of $\mathrm{Co}$ and 3 atoms of $\mathrm{O}$. We found that the Co atoms at the interface have enhanced magnetic moments compared to the values of the Co atoms of the bulk Co(fcc). This enhancement is in agreement with an X-ray magnetic circular dichroism study of $\mathrm{Co} / \mathrm{CoO}$ bilayer [9]. Small magnetic moments less than $0.1 \mu_{\mathrm{B}}$ are induced to the oxygen atoms at the interface. Calculations on $\mathrm{Co} / \mathrm{CoO}$ bilayers with different thickness of the layers are under investigation.

\section{Conclusions}

First-principles GGA+U calculations were employed to study the magnetic properties of $\mathrm{Co}_{x}$ and $\mathrm{Co}_{x-1} \mathrm{O}$ clusters, where $x=13,14$, and 19 , and on $\mathrm{Co} / \mathrm{CoO}$ bilayer. Our main conclusions are summarized below:

$\mathrm{Co}_{x}$ and $\mathrm{Co}_{x-1} \mathrm{O}$ clusters: The addition of the $\mathrm{O}$ atom in the Co cluster leads to an increase of the $\mu_{\text {Co }}$ values of O's adjacent Co atoms.

$\mathrm{Co} / \mathrm{CoO}$ bilayers: Small magnetic moment is induced by the $\mathrm{Co}$ atoms to the $\mathrm{O}$ atoms in the interface, while the $\mathrm{Co}$ atoms in the interface have enhanced magnetic moment compared to the corresponding values of the bulk Co (in agreement with the experiment), but smaller than those of the bulk CoO.

\section{Acknowledgment}

This research has been co-financed by the European Social Fund (EU) and Greek national funds through the Operational Program "Education and Lifelong Learning" in the framework of ARISTEIA I (Project COMANA/22).

\section{References}

1. C. Binns, J. A. Blackman, Metallic Nanoparticles, p. 277 (ed. J. A. Blackman, Elsevier 2009)

2. G. Rollmann, M. E. Gruner, A. Hucht, R. Meyer, P. Entel, M. L. Tiago, J. R. Chelikowsky, Phys. Rev. Lett. 99, 083402 (2007)

3. I. M. L. Billas, A. Châtelain, W. A. de Heer, Science 265, 1682 (1994)

4. G. Rollmann, S. Sahoo, A. Hucht, P. Entel, Phys. Rev. B 78, 134404 (2008)

5. S. Jin, T. Tiefel, M. McCormarck, R. Fastnacht, R. Rchen, Science 264, 413 (1994)

6. A. J. Millis, Nature 392, 147 (1998)

7. J. Bansmann, S. H. Baker, C. Binns, J. A. Blackman, J.-P. Bucher, J. Dorantes-Dávila, V. Dupuis, L. Favre, D. Kechrakos, A. Kleibert, K.-H. MeiwesBroer, G. M. Paster, A. Pérez, O. Toulemonde, K. N. Trohidou, J. Tuaillon, Y. Xie, Surf. Sci. Rep. 56, 189 (2005)

8. B. Yu. Yavorsky, I. Mertig, Phys. Rev. B 74, 174402 (2006).

9. A. M. Mulders, H. Loosvelt, A. Fraile Rodríguez, E. Popova, T. Konishi, K. Temst, O. Karis, D. Arvanitis, C. Van Haesendonck, J. Phys.: Condens. Matter 21, 124211 (2009)
10. W. Sucksmith, J. E. Thompson, Proc. Roy. Soc. London, Series A, Math. Phys. Sci. 225, 362 (1954)

11. D. Srikala, V. N. Singh, A. Banerjee, B. R. Mehta, S. Patnaik, J. Phys. Chem. C 112, 13882 (2008)

12. W. L. Roth, Phys. Rev. 1101333 (1958)

13. U. D. Wdowik and K. Parlinski, Phys. Rev. B 75, 104306 (2007)

14. R. W. G. Wyckoff, Crystal Structures, (2nd ed. Interscience Publishers, NY) 1, 7 (1963)

15. L. Marick, Phys. Rev. 49, 831 (1936)

16. J. Szczytko, N. Vaupotič, K. Madrak, P. Sznajder, E. Górecka, Phys. Rev. E 87, 033201 (2013)

17. R. W. Wyckoff, Crystal Structures, (2nd ed. Interscience Publishers, NY) 1, 85 (1963)

18. D. C. Kham, R. A. Erickson, Phys. Rev. B 1, 2243 (1970)

19. W. Jauch, M. Reehuis, H. J. Bleif, F. Kubanek, Phys. Rev. B 64, 052102 (2001)

20. J. van Elp, J. L. Wieland, H. Eskes, P. Kuiper, G. A. Sawatzky, F. M. F. de Groot, T. S. Turner, Phys. Rev. B 44, 6090 (1991)

21. H.-X. Deng, J. Li, S.-S. Li, J.-B. Xia, A. Walsh, S.H. Wei, Appl. Phys. Lett. 96, 162508 (2010)

22. A. Schrön, C. Rödl, F. Bechstedt, Phys. Rev. B 86, 115134 (2012)

23. T. Archer, R. Hanafin, S. Sanvito, Phys. Rev. B 78, 014431 (2008)

24. C. Rödl, F. Fuchs, J. Furthmüller, F. Bechstedt, Phys. Rev. B 79, 235114 (2009)

25. J. Nogués, I. K. Schuller, J. Magn. Magn. Mater. 192, 203 (1999)

26. A. Paul, N. Paul, J. Jutimoosik, R. Yimnirun, S. Rujirawat, B. Höpfner, I. Lauermann, M. LuxSteiner, S. Mattauch, P. Böni, Phys. Rev. B 87, 01431 (2013)

27. D. Le Roy, R. Morel, S. Pouget, A. Brenac, L. Notin, T. Crozes, W. Wernsdorfer, Phys. Rev. Lett. 107, 057204 (2011)

28. G. Kresse, J. Furthmüller, Comput. Mater. Sci. 6, 15 (1996)

29. G. Kresse, D. Joubert, Phys. Rev. B 59, 1758 (1999)

30. J. P. Perdew, Electronic structure of solids '91, p. 11 (ed. P. Ziesche and H. Esching, Akademie Verlag, Berlin 1991)

31. S. L. Dudarev, G. A. Botton, S. Y. Savrasov, C. J. Humphreys, A. P. Sutton, Phys. Rev. B 57, 1505 (1998)

32. J. P. Bucher, D. C. Douglass, L. A. Bloomfield, Phys. Rev. Lett. 66, 3052 (1991)

33. J. P. Chen, C. M. Sorensen, K. J. Klabunde, G. C. Hadjipanayis, Phys. Rev. B, 51, 11527 (1995)

34. T. Thomson, P. C. Riedi, S. Sankar, A. E. Berkowitz, J. Appl. Phys. 81, 5549 (1997)

35. S. Dennler, J. Morillo, G. M. Pastor, Surf. Science, 532-535, 334 (2003) 